\title{
BCJ numerators from differential operator of multidimensional residue
}

\author{
Gang Chen ${ }^{1,2,3, a}$, Tianheng Wang ${ }^{2,4,5}$ \\ ${ }^{1}$ Department of Physics, Zhejiang Normal University, Jinhua, China \\ 2 Department of Physics and Astronomy, Uppsala University, Uppsala, Sweden \\ ${ }^{3}$ Centre for Research in String Theory, School of Physics and Astronomy, Queen Mary University of London, London, UK \\ ${ }^{4}$ Department of Physics, Nanjing University, Nanjing, China \\ ${ }^{5}$ Institut für Physik, Humboldt-Universität zu Berlin, Berlin, Germany
}

Received: 7 September 2019 / Accepted: 31 December 2019 / Published online: 16 January 2020

(C) The Author(s) 2020

\begin{abstract}
In previous works, we devised a differential operator for evaluating typical integrals appearing in the Cachazo-He-Yuan (CHY) forms and in this paper we further streamline this method. We observe that at tree level, the number of free parameters controlling the differential operator depends solely on the number of external lines, after solving the constraints arising from the scattering equations. This allows us to construct a reduction matrix that relates the parameters of a higher-order differential operator to those of a lower-order one. The reduction matrix is theory-independent and can be obtained by solving a set of explicitly given linear conditions. The repeated application of such reduction matrices eventually transforms a given tree-level CHY-like integral to a prepared form. We also provide analytic expressions for the parameters associated with any such prepared form at tree level. We finally give a compact expression for the multidimensional residue for any CHY-like integral in terms of the reduction matrices. We adopt a dual basis projector which leads to the CHY-like representation for the non-local BernCarrasco-Johansson (BCJ) numerators at tree level in YangMills theory. These BCJ numerators are efficiently computed by the improved method involving the reduction matrix.
\end{abstract}

\section{Introduction}

Scattering amplitudes in a number of theories can be packaged in the compact expressions known as the Cachazo-HeYuan (CHY) forms [1-3]. The CHY forms are originally proposed for tree-level scattering amplitudes and later generalized to loop levels [4-13]. In the CHY form, the scattering amplitude is represented as a contour integral around the solutions to the scattering equations [1-3], which can

a e-mail: gang.chern@gmail.com be transformed to a polynomial form $[14,15]$. Such contour integrals can be evaluated using the integration rules and the cross-ratio method at tree and loop levels [16-19]. Systematic methods for computing these integrals are based on the analysis of multidimensional residues on the isolated solutions of the scattering equations. One method for computing multidimensional residues involving the Groebner basis or the H-basis is discussed in $[20,21]$. A useful Mathematica package for computing such residues is given in [22].

In $[23,24]$ Cheung, $\mathrm{Xu}$ and the current authors proposed a method for evaluating the $\mathrm{CHY}$ forms using a differential operator and studied the combinatoric properties of the scattering equations. This method bypasses the need for solving the scattering equations and leads to the analytic evaluation of a particular class of $\mathrm{CHY}$ forms, called the prepared forms. In this paper, we further streamline the method at tree level by relating a generic $\mathrm{CHY}$-like expression to a prepared form. A crucial observation in our approach is that the number of independent parameters appearing in such a differential operator is always $(n-3)$ ! where $n$ is the number of external lines, regardless of the order of the operator. As will become clear in later discussions, this observation allows us to develop a method that relates higher-order differential operators with lower-order ones, through the reduction matrices for each factor of the terms in the Pfaffian expansion. Our method is theory independent and it maintains the factorized form of $\mathrm{CHY}$ integrand. Due to the two advantages, the CHY integral is evaluated efficiently.

As a particular application of our method, we study the construction of the Bern-Carrasco-Johansson (BCJ) numerators [25] from the $\mathrm{CHY}$ forms. The color-kinematic duality is found to hold in a number of theories [25-36] and extensive studies have been dedicated to computing the BCJ numerators [37-41]. In $[38,42]$, the twistor string theory have been 
studied to extract the local Bern-Carrasco-Johansson (BCJ) [25] numerators. The CHY forms can also be used to study the BCJ numerators and in [43] the local BCJ numerators are constructed. In this paper, we extract the non-local BCJ numerators in the minimal basis at tree level from the $\mathrm{CHY}$ forms, by introducing a dual basis projector. This way the BCJ numerators also take CHY-like expressions and can be easily studied using the differential operator and the reduction matrix.

\section{Preliminary: review of differential operator method}

Here we briefly summarize our method for computing the multidimensional residue. Let $g_{1}, g_{2}, \ldots, g_{k}$ be homogeneous polynomials in complex variables $z_{1}, z_{2}, \ldots, z_{k}$. If their common zeros lie on a single isolated point $p$ (for homogeneous polynomials, the point $p$ is the origin), for a holomorphic function $\mathcal{R}\left(z_{i}\right)$ in a neighborhood of $p$, we conjecture that a differential operator $\mathbb{D}$ computes the residue of $\mathcal{R}$ at $p$ as follows

$$
\begin{aligned}
& \operatorname{Res}_{\left\{\left(g_{1}\right), \ldots,\left(g_{k}\right)\right\}, p}[\mathcal{R}] \equiv \oint \frac{d z_{1} \wedge \cdots \wedge d z_{k}}{g_{1} \ldots g_{k}} \mathcal{R} \\
& \quad=\left.\mathbb{D}^{(m)}[\mathcal{R}]\right|_{z_{i} \rightarrow 0},
\end{aligned}
$$

where $\mathbb{D}^{(m)}$ is a differential operator of order- $m$ and takes the following form,

$\mathbb{D}^{(m)}=\sum_{\left\{r_{i}\right\}_{m}} a_{r_{1}, r_{2}, \ldots, r_{k}} \partial^{r_{1}, r_{2}, \ldots, r_{k}}$

Here $\partial^{r_{1}, r_{2}, \ldots, r_{k}}=\left(\frac{\partial}{\partial z_{1}}\right)^{r_{1}}\left(\frac{\partial}{\partial z_{2}}\right)^{r_{2}} \cdots\left(\frac{\partial}{\partial z_{k}}\right)^{r_{k}}$ and $r_{i}$ 's are non-negative integers satisfying the Frobenius equation $\sum_{i=1}^{k} r_{i}=m \equiv \sum_{i=1}^{k} \operatorname{deg}\left(g_{i}\right)-k$. The coefficients $a_{r_{1}, r_{2}, \ldots, r_{k}}$ are constants independent of $z_{i}$ 's, determined uniquely by two sets of constraints arising from: 1 . the local duality theorem [44] and 2. the intersection number of the divisors $D_{i}=\left(g_{i}\right)$. Detailed discussions on these constraints can be found in [23]. This conjecture applies to any such multidimensional residues around an isolated pole.

The CHY form for a tree-level scattering amplitude or a loop-level integrand is an integral on a Riemann sphere completely localized by the scattering equations. Equivalently, it is a multi-dimensional residue around the common zeros of the scattering equations. The tree-level scattering equations for $n$ external particles read

$\sum_{j \neq i} \frac{k_{i} \cdot k_{j}}{\sigma_{i}-\sigma_{j}}=0, \quad i \in[2, n-2]$, where we have already taken care of the $S L(2, \mathbb{C})$ conformal symmetry by fixing $\sigma_{1} \rightarrow 0, \sigma_{n-1} \rightarrow 1, \sigma_{n} \rightarrow \infty$. A simple transformation found in $[14,15]$ takes $(3)$ to the polynomial ones

$$
\begin{aligned}
h_{t} & =\left.\left(\sum_{2 \leqslant i_{1}<i_{2}<\cdots<i_{t} \leqslant n-1} s_{i_{1} i_{2} \ldots i_{t} n} \sigma_{i_{1}} \ldots \sigma_{i_{t}}\right)\right|_{\sigma_{n-1} \rightarrow \sigma_{0}}, \\
& t \in[1, n-3]
\end{aligned}
$$

where $s_{i_{1} \ldots i_{t} n}=\frac{1}{2}\left(k_{i_{1}}+\cdots+k_{i_{t}}+k_{n}\right)^{2}$. Here we have introduced an auxiliary variable $\sigma_{0}$, which fomally renders the polynomials homogeneous for the above differential operator to apply. At $\sigma_{0} \rightarrow 1$, the two versions of scattering equations, (3) and (4), are equivalent. ${ }^{1}$ The Jacobian of the above transformation is given by a Vandermonde determinant $J_{n}(\sigma)=\prod_{1 \leq r<t \leq n-1}\left(\sigma_{t}-\sigma_{r}\right)$.

Adopting the polynomial scattering equations, a tree-level $n$-point amplitude is schematically given by a combination of the following CHY-like integrals

$$
\begin{aligned}
\mathcal{I}_{n}\left(P, h_{0}\right)= & \oint_{h_{1}=\cdots=h_{n-3}=\sigma_{0}-1=0} \\
& \frac{d \sigma_{2} \wedge \cdots \wedge d \sigma_{n-2} \wedge d \sigma_{0}}{h_{1} \ldots h_{n-3}\left(\sigma_{0}-1\right)} \frac{P(\sigma)}{h_{0}(\sigma)},
\end{aligned}
$$

where the integrand is a rational function specific to the underlying theory. Its explicit expressions in different contexts can be found in [1-3]. For our purpose, we only note that $h_{0}(\sigma)$ is a homogeneous polynomial and factorizes into products of degree-one polynomials. In addition to the $(n-3)$ polynomial scattering equations, $\sigma_{0}-1=0$ is also imposed to localize the auxiliary variable.

The global residue theorem allows us to consider the residue around the solution of $h_{1}=\cdots=h_{n-3}=h_{0}=0$ instead $^{2}$

$$
\begin{aligned}
\mathcal{I}_{n}\left(P, h_{0}\right)=- & \oint_{\substack{h_{1}=\cdots=h_{n-3}=h_{0}=0\\
}} \\
& \frac{d \sigma_{2} \wedge \cdots \wedge d \sigma_{n-2} \wedge d \sigma_{0}}{h_{1} \ldots h_{n-3} h_{0}(\sigma)} \frac{P(\sigma)}{\left(\sigma_{0}-1\right)} .
\end{aligned}
$$

The aforementioned differential operator then applies to (6) as follows

$\mathcal{I}_{n}\left(P, h_{0}\right)=-\left.\left[\mathbb{D}_{h_{0}}^{(m)} \frac{P(\sigma)}{\left(\sigma_{0}-1\right)}\right]\right|_{\sigma \rightarrow 0}$,

\footnotetext{
1 The polynomial scattering equations are equivalent to the original ones at tree level. At loop levels, there are extra solutions, which is beyond the scope of this paper.

2 Poles at infinity can in principle exist. Detailed discussions on poles at infinity are given in [23].
} 
where $\sigma \rightarrow 0$ is simply a shorthand for $\sigma_{r} \rightarrow 0, r \in$ $\{2, \ldots, n-2,0\}$. Namely all $\sigma_{i}$ 's are taken to zero after the action of the differential operator. The differential operator $\mathbb{D}_{h_{0}}^{(m)}$ takes the form given in (2) with the parameters $a_{r_{2}, r_{3}, \ldots, r_{n-2}, r_{0}}$ and

$$
\begin{gathered}
\partial^{r_{2}, r_{3}, \ldots, r_{n-2}, r_{0}}=\left(\frac{\partial}{\partial \sigma_{2}}\right)^{r_{2}}\left(\frac{\partial}{\partial \sigma_{3}}\right)^{r_{3}} \\
\ldots\left(\frac{\partial}{\partial \sigma_{n-2}}\right)^{r_{n-2}}\left(\frac{\partial}{\partial \sigma_{0}}\right)^{r_{0}} .
\end{gathered}
$$

The parameters $a_{r_{2}, r_{3}, \ldots, r_{n-2}, r_{0}}$ are determined by the polynomial scattering equations $h_{j}(j \in[1, n-3])$ and $h_{0}$. As the scattering equations are universal for all CHY-like integrals, we only specify $h_{0}$ in the labels of the differential operator. The order of this operator is again $m=0+1+\cdots+(n-$ 4) $+d_{h_{0}}-1$, with $d_{h_{0}}$ denoting the degree of $h_{0}$. We note that the scattering equations $h_{j}=0(j \in[1, n-3])$ and $\sigma_{0}-1=0$ have multiple common solutions. Namely (5) has multiple poles. The differential operator in (7) gives the sum of (5) evaluated at each solution, by the global residue theorem. For details, see [23]. In particular, when $h_{0}=\sigma_{i}$, the CHY-like integral is studied in [24] and the corresponding differential operator is worked out analytically.

\section{Reduction matrix and evaluation of CHY integrals}

In this section, we propose a method for relating two differential operators associated with two CHY-like integrals. To be more precise, the two CHY-like integrals of the form (5) share the numerator $P(\sigma)$ and their respective $h_{0}$ and $h_{0}^{\prime}$ are related as $h_{0}(\sigma)=h_{0}^{\prime}(\sigma) q(\sigma)$ with $q(\sigma)$ being also a polynomial. In this case, the $a$-coefficients in the two corresponding differential operators can be related by a matrix, which we call the reduction matrix. This leads to a systematic evaluation of any tree-level CHY-like integral, which is given in a factorized form.

\subsection{Canonical coefficients in differential operator}

Consider the differential forms below

$\Gamma=\frac{P(\sigma) d \sigma_{2} \wedge \cdots \wedge d \sigma_{n-2} \wedge d \sigma_{0}}{h_{1} \ldots h_{n-3} h_{0}}$,

whose residue at the origin is the same as the CHY-like integral of the form (5). Recall that the corresponding differential operator $\mathbb{D}_{h_{0}}^{(m)}$ takes the form of (2) as follows

$\mathbb{D}_{h_{0}}^{(m)}=\sum_{\{r\}_{m}} a_{r_{2}, \ldots, r_{n-2}, r_{0}} \partial^{r_{2}, \ldots, r_{n-2}, r_{0}}$.
Since the polynomial scattering equations $\left\{h_{1}, \ldots, h_{n-3}\right\}$ are universal, we can always solve the local duality conditions [23] arising from these polynomials first. These conditions read

$\mathbb{D}_{h_{0}}^{(m)}\left[q_{j}(\sigma) h_{j}(\sigma)\right]=0, \quad j=1,2, \ldots n-3$,

where $q_{j}(\sigma)$ scans over all the monomials in $\sigma$ 's of the degree $\operatorname{deg}\left(q_{j}\right)=m-j$. Substituting (9) into (10), we have

$$
\begin{gathered}
\sum_{i_{1}<i_{2}<\cdots<i_{t}}\left[\prod_{l=2}^{n-1}\left(r_{l}+v_{l}\right) !\right] \\
s_{i_{1} i_{2} \ldots i_{t} i_{n}} a_{r_{2}+v_{2}, r_{3}+v_{3}, \ldots, r_{n-2}+v_{n-2}, r_{0}+v_{0}}=0,
\end{gathered}
$$

where we always identify $r_{n-1}=r_{0}$ and $v_{n-1}=v_{0}$. The summation is taken over the subsets $\left\{i_{1}, \ldots, i_{t}\right\} \subset$ $\{2, \ldots, n-1\}$ with $t \in[1, n-3]$. Here $r_{s}$ 's are non-negative integers and $v_{l}$ 's are defined as

$v_{l}=\left\{\begin{array}{ll}1, & \text { if } l \in\left\{i_{1}, i_{2}, \ldots, i_{t}\right\} \\ 0, & \text { if } l \notin\left\{i_{1}, i_{2}, \ldots, i_{t}\right\}\end{array}\right.$.

For a given $n$, the number of the $a$-coefficients and the number of local duality conditions both grow as $d_{h_{0}}$ increases. However, we observe that the number of independent $a$ coefficients after solving the Eq. (11) is always $(n-3)$ !, regardless of $m .^{3}$ This allows us to choose $(n-3) ! a$ coefficients as a basis and expand the rest on this basis.

For the purpose of this paper, we find a particularly convenient basis choice as follows,

$\left\{a_{\gamma(0), \ldots, \gamma(n-4),\left(d_{h_{0}}-1\right)} \mid \gamma \in S_{n-3}\right\}$,

where $S_{n-3}$ denotes the permutations. Throughout this paper, the $a$-coefficients in the above set is called the canonical coefficients. The differential operator can then be rewritten only in the canonical coefficients

$\mathbb{D}_{h_{0}}^{(m)}=\sum_{i=1}^{(n-3) !} a_{\gamma_{i}(0), \ldots, \gamma_{i}(n-4), d_{h_{0}}-1} \mathcal{D}_{i}^{(m)}$,

where each $\gamma_{i}$ denotes a different permutation. The noncanonical $a$-coefficients are expanded into the canonical ones

$a_{r_{2}, \ldots, r_{n-3}, r_{0}}=\sum_{i=1}^{(n-3) !} c_{i}^{r_{2}, \ldots, r_{n-3}, r_{0}} a_{\gamma_{i}(0), \ldots, \gamma_{i}(n-4),\left(d_{h_{0}}-1\right)}$,

\footnotetext{
3 This is an observation from a large number of examples, both analytic and numeric. We don't have a proof for this observation at the moment.
} 
with the coefficients $c_{i}^{r_{2}, \ldots, r_{n-3}, r_{0}}$ obtained by solving (11). Collecting the canonical coefficients, we have

$$
\begin{aligned}
\mathcal{D}_{i}^{(m)}= & \partial^{\gamma_{i}(0), \ldots, \gamma_{i}(n-4),\left(d_{h_{0}}-1\right)} \\
& +\sum_{\substack{\sum_{\left\{r_{j}\right\} \notin S_{n-3}} r_{j}=m, r_{n}}} c_{i}^{r_{2}, \ldots, r_{n-3}, r_{0}} \partial^{r_{2}, \ldots, r_{n-2}, r_{0}} .
\end{aligned}
$$

In (14), the differential operator $\mathbb{D}_{h_{0}}^{(m)}$ is expanded into the basis spanned by $\mathcal{D}_{i}^{(m)}, i \in[1,(n-3) !]$. We note that $\mathcal{D}_{i}^{(m)}$ 's are determined solely by the order $m$ and the scattering equations, independent of the actual form of $h_{0}$.

\subsection{Reduction matrix}

We now study the relation between the higher- and lowerorder operators. Consider the meromorphic forms $\Gamma$ and $\Gamma^{\prime}$ below

$$
\begin{gathered}
\Gamma=\frac{P(\sigma) d \sigma_{2} \wedge \cdots \wedge d \sigma_{n-2} \wedge d \sigma_{0}}{h_{1} \ldots h_{n-3} h_{0}}, \\
\Gamma^{\prime}=\frac{P(\sigma) d \sigma_{2} \wedge \cdots \wedge d \sigma_{n-2} \wedge d \sigma_{0}}{h_{1} \ldots h_{n-3} h_{0}^{\prime}},
\end{gathered}
$$

where $h_{0}(\sigma)=h_{0}^{\prime}(\sigma) q(\sigma)$ with $q(\sigma)$ also being a polynomial of degree $d_{q}$. Let $\mathbb{D}_{h_{0}}^{(m)}$ and $\mathbb{D}_{h_{0}^{\prime}}^{\left(m-d_{q}\right)}$ denote their corresponding differential operators respectively. For an arbitrary homogeneous polynomial $P(\sigma)$ of degree $\operatorname{deg}(P) \leqslant m-d_{q}$, we must have

$$
\left.\left[\mathbb{D}_{h_{0}}^{(m)} \frac{q(\sigma) P(\sigma)}{\sigma_{0}-1}\right]\right|_{\sigma \rightarrow 0}=\left.\left[\mathbb{D}_{h_{0}^{\prime}}^{\left(m-d_{q}\right)} \frac{P(\sigma)}{\sigma_{0}-1}\right]\right|_{\sigma \rightarrow 0} .
$$

Plugging in the solutions of the respective non-canonical $a$ coefficients on both sides and expressing both differential operators in terms of their canonical coefficients only, the equation above becomes

$$
\begin{aligned}
& \left.\sum_{i=1}^{(n-3) !} a_{\gamma_{i}(0), \ldots, \gamma_{i}(n-4), d_{h_{0}}-1}\left[\mathcal{D}_{i}^{(m)} \frac{q(\sigma) P(\sigma)}{\sigma_{0}-1}\right]\right|_{\sigma \rightarrow 0} \\
& =\left.\sum_{i=1}^{(n-3) !} a_{\gamma_{i}(0), \ldots, \gamma_{i}(n-4), d_{h_{0}}-d_{q}-1}\left[\mathcal{D}_{i}^{\left(m-d_{q}\right)} \frac{P(\sigma)}{\sigma_{0}-1}\right]\right|_{\sigma \rightarrow 0} .
\end{aligned}
$$

For this equation to hold for an arbitrary $P(\sigma)$, the coefficients of the surviving derivatives $\partial^{r_{2}, \ldots, r_{n-3}, r_{0}} P$ with $r_{2}+$ $\cdots+r_{n-3}+r_{0}<\operatorname{deg}(P)$ must be the same on both sides. This leads to linear relations between the two sets of canonical $a$-coefficients, which can be written in the matrix form

$$
\begin{gathered}
\left(\begin{array}{c}
\vdots \\
a_{\gamma(0), \ldots, \gamma(n-4), d_{h_{0}}-1} \\
\vdots
\end{array}\right) \\
=M_{q(\sigma)}^{(n, m)}\left(\begin{array}{c}
a_{\gamma(0), \ldots, \gamma(n-4), d_{h_{0}}-d_{q}-1} \\
\vdots
\end{array}\right) .
\end{gathered}
$$

We name the matrix $M_{q(\sigma)}^{(n, m)}$ the reduction matrix. The reduction matrix depends only on the factor $q(\sigma)$ and the orders of the differential operators while it knows nothing about the specific expression of the factor $h_{0}^{\prime}$. We note that although the reduction matrix depends on $q(\sigma)$, its entries are only functions of momenta.

The reduction process can be performed repeatedly. Typically $h_{0}$ in a CHY form is completely factorized as $h_{0}=$ $q^{(1)} q^{(2)} \ldots q^{\left(d_{h_{0}}\right)}$, where each $q^{(r)}=\sigma_{i}-\sigma_{j}$ is a degreeone polynomial in $\sigma$ 's. As a result, the canonical coefficients in $\mathbb{D}_{h_{0}}^{(m)}$ can eventually be related to those in an operator of order $m_{0} \equiv m-\left(d_{h_{0}}-1\right)$. For such a degree-one $q=\sigma_{i}-\sigma_{j}$, it is easy to check that (19) yields a simple relation between reduction matrices $M_{q}^{(n, m)}=\frac{1}{m-m_{0}} M_{q}^{\left(n, m_{0}+1\right)}$. For notational brevity, we define $M_{q}^{\left(n, m_{0}+1\right)} \equiv M_{q}^{(n)}$. Hence we have

$$
\begin{aligned}
& \left(\begin{array}{c}
\vdots \\
a_{\gamma(0), \ldots, \gamma(n-4), d_{h_{0}}-1}
\end{array}\right) \\
& \vdots \\
& =\frac{M_{q^{(1)}}^{(n)} M_{q^{(2)}}^{(n)} \ldots M_{q^{\left(d_{h_{0}}-1\right)}}^{(n)}}{\left(d_{h_{0}}-1\right) !}\left(\begin{array}{c}
\vdots \\
a_{\gamma(0), \ldots, \gamma(n-4), 0} \\
\vdots
\end{array}\right) .
\end{aligned}
$$

We note that the ordering of these $q^{(r)}$ factors do not affect the eventual evaluation of the CHY-like integral. The choice of $\left(d_{h_{0}}-1\right)$ factors for the reduction process is also irrelevant, although certain choices might be more convenient in particular cases.

The inverse of the reduction matrix is linear in the variables in the subscript, namely ${ }^{4}$

\footnotetext{
$\overline{4}$ The relation (19) can be schematically rewritten as $L_{q} \cdot \mathbf{a}_{h_{h_{0}}-1}=$ $R \cdot \mathbf{a}_{d_{h_{0}}-d_{q}-1}$ where $\mathbf{a}_{d_{h_{0}}-1}$ and $\mathbf{a}_{d_{h_{0}}-d_{q}-1}$ are the two column vectors on the left and right sides of (20) respectively. $L_{q}$ and $R$ are matrices following directly from (19) and we note the matrix $R$ is independent of $q$. Hence $M_{q}=L_{q}^{-1} \cdot R$. For $q=\sigma_{r_{1}}-\sigma_{r_{2}},(19)$ yields $L_{\sigma_{r_{1}}-\sigma_{r_{2}}}=$ $L_{\sigma_{r_{1}}}-L_{\sigma_{r_{2}}}$. Moreover, since $M_{\sigma_{r_{1}}-\sigma_{r_{2}}}=L_{\sigma_{r_{1}}-\sigma_{r_{2}}}^{-1} \cdot R$ and $M_{\sigma_{r_{i}}}=$ $L_{\sigma_{r_{i}}}^{-1} \cdot R, i=1,2$, taking the inverse of the three reduction matrices, we obtain the relation (22).
} 


$$
\begin{aligned}
& M_{\sigma_{r_{1}}-\sigma_{r_{2}}}^{(n)} \\
& \quad=\left(\left(M_{\sigma_{r_{1}}}^{(n)}\right)^{-1}-\left(M_{\sigma_{r_{2}}}^{(n)}\right)^{-1}\right)^{-1} .
\end{aligned}
$$

Hence we only need to construct $\left(M_{\sigma_{r}}^{(n)}\right)^{-1}$, where $r \in$ $\{2, \ldots, n-2,0\}$. For $q(\sigma)=\sigma_{0}$, it is easy to verify that the reduction matrix $M_{\sigma_{0}}^{(n)}$ is the $(n-3)$ !-dimensional identity matrix. For $q(\sigma)=\sigma_{r}$, the Eq. (19) reads

$$
\begin{aligned}
& (\gamma(r-2)+1) a_{\gamma(0), \ldots, \gamma(r-3), \gamma(r-2)+1, \gamma(r-1), \ldots, \gamma(n-4), 0} \\
& \quad=a_{\gamma(0), \ldots, \gamma(n-4), 0},
\end{aligned}
$$

which holds for any $\gamma \in S_{n-3}$. The $a$-coefficient on the lefthand side above is not a canonical coefficient and can be rewritten in terms of the canonical ones as follows

$$
\begin{aligned}
& a_{\gamma(0), \ldots, \gamma(r-3), \gamma(r-2)+1, \gamma(r-1), \ldots, \gamma(n-4), 0} \\
& =\sum_{j=1}^{(n-3) !}\left(c_{j}^{\gamma(0), \ldots, \gamma(r-3), \gamma(r-2)+1, \gamma(r-1), \ldots, \gamma(n-4), 0} a_{\gamma_{j}(0), \ldots, \gamma_{j}(n-4), 1}\right),
\end{aligned}
$$

where the coefficients $c$ 's are defined in (15) and are solely determined by (11). From this equation we read out the elements of $\left(M_{\sigma_{r}}^{(n)}\right)^{-1}$ for $r \in[2, n-2]$ as follows

$$
\begin{aligned}
& \left(M_{\sigma_{r}}^{(n)}\right)_{i j}^{-1} \\
& \quad=\left(\gamma_{i}(r-2)+1\right) c_{j}^{\gamma_{i}(0), \ldots, \gamma_{i}(r-3), \gamma_{i}(r-2)+1, \gamma_{i}(r-1), \ldots, \gamma_{i}(n-4), 0} .
\end{aligned}
$$

Using the reduction matrices repeatedly, any CHY-like integral with a completely factorized $h_{0}$ can be related to one with $h_{0}^{\prime}=\sigma_{r} .^{5}$ The latter is the so-called prepared form and in [24] such CHY-like integrals with the one-loop scattering equations are studied. Here we repeat the exercise for the tree-level scattering equations. Let $a^{\left(\sigma_{r}\right)}$ denote the $a$ coefficients in the differential operator associated with the tree-level prepared form with $h_{0}=\sigma_{r}$. We obtain the following analytical expressions for the canonical ones

$$
\begin{aligned}
& \mathcal{I}_{n}\left(P, h_{0}\right)=-\left.\left[\mathbb{D}_{h_{0}}^{(m)} \frac{P(\sigma)}{\left(\sigma_{0}-1\right)}\right]\right|_{\sigma \rightarrow 0}=\frac{-1}{\left(d_{h_{0}}-1\right) !} \\
& \quad \times \sum_{i, j=1}^{(n-3) !}\left(M_{q^{(1)}}^{(n)} \ldots M_{q^{\left(d_{h_{0}}-1\right)}}^{(n)}\right)_{i j} a_{\gamma_{j}(0), \ldots, \gamma_{j}(n-4), 0}^{\left(\sigma_{r}\right)} \\
& \\
& {\left.\left[\mathcal{D}_{i}^{(m)} \frac{P(\sigma)}{\left(\sigma_{0}-1\right)}\right]\right|_{\sigma \rightarrow 0} .}
\end{aligned}
$$

\subsection{Examples}

Here we consider a couple of examples in detail to demonstrate the evaluation of tree-level CHY integrals, using the reduction matrix discussed above.

At four points, we have only one scattering equation after gauge fixing $\sigma_{1} \rightarrow 0, \sigma_{3} \rightarrow 1, \sigma_{4} \rightarrow \infty$. We consider the integral below as a simple example

$$
\begin{aligned}
\mathcal{I}_{4}\left(1, \sigma_{2}-1\right) & =\oint_{h_{1}=0} \frac{d \sigma_{2}}{h_{1}} \frac{1}{\sigma_{2}-1} \\
& =\oint_{h_{1}=\sigma_{0}-1=0} \frac{d \sigma_{2} \wedge d \sigma_{0}}{h_{1}\left(\sigma_{0}-1\right)} \frac{1}{\left(\sigma_{2}-\sigma_{0}\right) \sigma_{0}}
\end{aligned}
$$

where in the second equal sign we have homogenized the scattering equation and the original denominator of the integrand. We have also used the trick of multiplying $\frac{1}{\sigma_{0}}$ to the integrand for the later use of the prepared form. The homogenized polynomial scattering equation reads

$h_{1}=s_{13} \sigma_{2}+s_{12} \sigma_{0}$.

We use the global residue theorem to change the integral contour around the solutions of $h_{1}=\left(\sigma_{2}-\sigma_{0}\right) \sigma_{0}=0$

$$
\mathcal{I}_{4}\left(1, \sigma_{2}-1\right)=-\oint_{h_{1}=\left(\sigma_{2}-\sigma_{0}\right) \sigma_{0}=0} \frac{d \sigma_{2} \wedge d \sigma_{0}}{h_{1}\left(\sigma_{2}-\sigma_{0}\right) \sigma_{0}} \frac{1}{\left(\sigma_{0}-1\right)}
$$

$a_{\gamma(0), \ldots, \gamma(n-4), 0}^{\left(\sigma_{r}\right)}=\left\{\begin{array}{cc}\frac{\operatorname{sgn}(\gamma)(n-3) !}{\left.\left(\partial^{\gamma(0)+1, \ldots, \gamma(r-3)+1,0, \gamma(r-1)+1, \ldots, \gamma(n-4)+1,1}\left(h_{1} h_{2} \ldots h_{n-3}\right)\right)\right|_{\sigma \rightarrow 0},} & \text { for } \gamma(r-2)=0 \\ 0 & \text { for others }\end{array}\right.$,

$a_{\gamma(0), \ldots, \gamma(n-4), 0}^{\left(\sigma_{0}\right)}=\frac{\operatorname{sgn}(\gamma)(n-3) !}{\left.\left(\partial^{\gamma(0)+1, \ldots, \gamma(n-4)+1,0}\left(h_{1} h_{2} \ldots h_{n-3}\right)\right)\right|_{\sigma \rightarrow 0}}$,

where $\operatorname{sgn}(\gamma)$ denotes the signature of the permutation $\gamma$. With the reduction matrices and the $a$-coefficients above, a generic CHY integral (7) can be evaluated straightforwardly

\footnotetext{
5 Even if $h_{0}$ does not have a factor $\sigma_{r}$, one can always multiply $\frac{\sigma_{r}}{\sigma_{r}}$ or $\frac{1}{\sigma_{0}}$ to the integrand and apply the reduction process to other factors.
}

This integral is then given by the action of a differential operator as follows

$$
\mathcal{I}_{4}\left(1, \sigma_{2}-1\right)=-\left.\left[\mathbb{D}_{\left(\sigma_{2}-\sigma_{0}\right) \sigma_{0}}^{(1)} \frac{1}{\left(\sigma_{0}-1\right)}\right]\right|_{\sigma \rightarrow 0}
$$


where $\mathbb{D}_{\left(\sigma_{2}-\sigma_{0}\right) \sigma_{0}}^{(1)}=a_{1,0} \partial^{1,0}+a_{0,1} \partial^{0,1}$ and $\partial^{r_{2}, r_{0}}=$ $\left(\frac{\partial}{\partial \sigma_{2}}\right)^{r_{2}}\left(\frac{\partial}{\partial \sigma_{0}}\right)^{r_{0}}$. The non-canonical $a_{1,0}$ is related to the canonical $a_{0,1}$ via (11) and we have

$\mathbb{D}_{\left(\sigma_{2}-\sigma_{0}\right) \sigma_{0}}^{(1)}=a_{0,1} \mathcal{D}_{1}^{(1)}, \quad$ with $\quad \mathcal{D}_{1}^{(1)}=\partial^{0,1}-\frac{s_{12}}{s_{13}} \partial^{1,0}$.

The reduction matrix $M_{\sigma_{2}-\sigma_{0}}^{(4)}$ can be obtained from (22) and (25) directly, which relates $a_{0,1}$ above to $a_{0,0}^{\left(\sigma_{0}\right)}$ given by (26). We have

$$
\begin{aligned}
M_{\sigma_{2}-\sigma_{0}}^{(4)} & =-\frac{s_{13}}{\left(s_{12}+s_{13}\right)}, \\
a_{0,0}^{\left(\sigma_{0}\right)} & =\left.\left[\frac{1}{\partial^{1,0}\left(h_{1}\right)}\right]\right|_{\sigma \rightarrow 0}=\frac{1}{s_{13}} .
\end{aligned}
$$

Hence the integral reads simply

$$
\begin{aligned}
\mathcal{I}_{4}\left(1, \sigma_{2}-1\right) & =-\left.M_{\sigma_{2}-\sigma_{0}}^{(4)} a_{0,0}^{\left(\sigma_{0}\right)}\left[\mathcal{D}_{1}^{(1)} \frac{\sigma_{0}}{\left(\sigma_{0}-1\right)}\right]\right|_{\sigma \rightarrow 0} \\
& =-\frac{1}{s_{12}+s_{13}}
\end{aligned}
$$

At five points, we consider below a typical CHY-like integral with a double pole and a nontrivial numerator

$$
\begin{aligned}
& \mathcal{I}_{5}\left(\left(\sigma_{2}-1\right) \sigma_{3},\left(\sigma_{3}-1\right)^{2} \sigma_{2}\right) \\
& \quad=\oint_{\substack{h_{1}=h_{2}=0 \\
\sigma_{0}-1=0}} \frac{d \sigma_{2} \wedge d \sigma_{3} \wedge d \sigma_{0}}{h_{1} h_{2}\left(\sigma_{0}-1\right)} \frac{\left(\sigma_{2}-1\right) \sigma_{3}}{\left(\sigma_{3}-\sigma_{0}\right)^{2} \sigma_{2}},
\end{aligned}
$$

where we have homogenized the scattering equations and the denominator $\left(\sigma_{3}-1\right)^{2} \sigma_{2}$ with the auxiliary variable $\sigma_{0}$. The numerator does not need to be homogenized for the application of our method. The homogenized polynomial scattering equations are

$$
\begin{aligned}
& h_{1}=s_{134} \sigma_{2}+s_{124} \sigma_{3}+s_{123} \sigma_{0} \\
& h_{2}=s_{14} \sigma_{2} \sigma_{3}+s_{13} \sigma_{2} \sigma_{0}+s_{12} \sigma_{3} \sigma_{0} .
\end{aligned}
$$

After using the global residues theorem, the integral contour is changed to the circle around the solutions of $h_{1}=h_{2}=$ $\left(\sigma_{3}-\sigma_{0}\right)^{2} \sigma_{2}=0$. This integral is then given by the action of the following differential operator

$$
\begin{aligned}
& \mathcal{I}_{5}\left(\left(\sigma_{2}-1\right) \sigma_{3},\left(\sigma_{3}-1\right)^{2} \sigma_{2}\right) \\
& \quad=-\left.\left[\mathbb{D}_{\left(\sigma_{3}-\sigma_{0}\right)^{2} \sigma_{2}}^{(3)} \frac{\left(\sigma_{2}-1\right) \sigma_{3}}{\left(\sigma_{0}-1\right)}\right]\right|_{\sigma \rightarrow 0} .
\end{aligned}
$$

The differential operator takes the form below

$$
\mathbb{D}_{\left(\sigma_{3}-\sigma_{0}\right)^{2} \sigma_{2}}^{(3)}=a_{0,0,3} \partial^{0,0,3}+a_{1,1,1} \partial^{1,1,1}+a_{0,1,2} \partial^{0,1,2}+\cdots,
$$

where $\partial^{r_{2}, r_{3}, r_{0}}=\left(\frac{\partial}{\partial \sigma_{2}}\right)^{r_{2}}\left(\frac{\partial}{\partial \sigma_{3}}\right)^{r_{3}}\left(\frac{\partial}{\partial \sigma_{0}}\right)^{r_{0}}$ and we have only written out the terms that have nonzero contributions. Here (11) gives the following equations

$$
\begin{array}{r}
6 s_{45} a_{0,0,3}+2 s_{35} a_{0,1,2}+2 s_{25} a_{1,0,2}=0 \\
s_{235} a_{1,1,1}+2 s_{345} a_{0,1,2}+2 s_{245} a_{1,0,2}=0 .
\end{array}
$$

The canonical coefficients here are $\left\{a_{0,1,2}, a_{1,0,2}\right\}$ and hence we have

$\mathbb{D}_{\left(\sigma_{3}-\sigma_{0}\right)^{2} \sigma_{2}}^{(3)}=a_{0,1,2} \mathcal{D}_{1}^{(3)}+a_{1,0,2} \mathcal{D}_{2}^{(3)}$

where

$\mathcal{D}_{1}^{(3)}=-\frac{s_{35}}{3 s_{45}} \partial^{0,0,3}-\frac{2 s_{12}}{s_{14}} \partial^{1,1,1}+\partial^{0,1,2}+\cdots$

$\mathcal{D}_{2}^{(3)}=-\frac{s_{2,5}}{3 s_{45}} \partial^{0,0,3}-\frac{2 s_{13}}{s_{14}} \partial^{1,1,1}+\partial^{1,0,2}+\cdots$.

Applying the reduction matrix for the factor $\sigma_{3}-\sigma_{0}$ twice, the above canonical coefficients $\left\{a_{0,1,2}, a_{1,0,2}\right\}$ are related to the canonical coefficients $\left\{a_{0,1,0}^{\left(\sigma_{2}\right)}, a_{1,0,0}^{\left(\sigma_{2}\right)}\right\}$. Hence we only need to construct $M_{\sigma_{3}-\sigma_{0}}^{(5)}$. The reduction matrix for $\sigma_{0}$ is just two dimensional identity matrix. Recall the linearity property (22) and that $M_{\sigma_{0}}^{(5)}$ is simply a two-dimensional identity matrix. The only nontrivial ingredient here is $\left(M_{\sigma_{3}}^{(5)}\right)^{-1}$. Recall that $M_{\sigma_{3}}^{(5)}$ is defined to relate an order-2 differential operator to an order- 1 one. For $q=\sigma_{3}$, the relation (23) reads

$a_{0,1,0}=2 a_{0,2,0}, \quad a_{1,0,0}=a_{1,1,0}$.

Moreover, the local duality conditions (11) for the $a$ coefficients in the order- 2 differential operator read

$$
\begin{aligned}
2 s_{35} a_{0,2,0}+s_{25} a_{1,1,0}+s_{45} a_{0,1,1} & =0, \\
s_{235} a_{1,1,0}+s_{345} a_{0,1,1}+s_{245} a_{1,0,1} & =0 .
\end{aligned}
$$

Plugging (42) in (43) we obtain

$$
\left(\begin{array}{l}
a_{0,1,0} \\
a_{1,0,0}
\end{array}\right)=\left(\begin{array}{cc}
-\frac{s_{35}}{s_{45}} & -\frac{s_{25}}{s_{45}} \\
\frac{s_{12} s_{35}}{s_{13} s_{45}} & \frac{s_{12} s_{25}-s_{14} s_{45}}{s_{13} s_{45}}
\end{array}\right)\left(\begin{array}{l}
a_{0,1,1} \\
a_{1,0,1}
\end{array}\right),
$$

where the matrix is $\left(M_{\sigma_{3}}^{(5)}\right)^{-1}$. With this matrix obtained, we can compute $M_{\sigma_{3}-\sigma_{0}}^{(5)}$ straightforwardly. 
The canonical coefficients $\left\{a_{0,1,0}^{\left(\sigma_{2}\right)}, a_{1,0,0}^{\left(\sigma_{2}\right)}\right\}$ are again given by (26) as follows

$$
a_{0,1,0}^{\left(\sigma_{2}\right)}=\frac{2}{\left.\left(\partial^{0,2,1}\left(h_{1} h_{2}\right)\right)\right|_{\sigma \rightarrow 0}}=\frac{1}{s_{12} s_{124}}, \quad a_{1,0,0}^{\left(\sigma_{2}\right)}=0 .
$$

Hence we have

$$
\begin{aligned}
\mathcal{I}_{5}= & -\frac{1}{2} \sum_{i, j=1}^{2}\left(M_{\sigma_{3}-\sigma_{0}}^{(5)} M_{\sigma_{3}-\sigma_{0}}^{(5)}\right)_{i, j} a_{\gamma_{i}(0), \gamma_{i}(1), 0}^{\left(\sigma_{2}\right)} \\
& \times\left.\left[\mathcal{D}_{j}^{(3)} \frac{1}{\left(\sigma_{0}-1\right)}\right]\right|_{\sigma \rightarrow 0} \\
= & \frac{s_{234} s_{14}+s_{12} s_{24}+s_{234} s_{24}}{s_{12} s_{234} s_{34}^{2}}
\end{aligned}
$$

where $\gamma_{i} \in S_{2}$.

\section{Gauge invariant BCJ numerators of Yang-Mills}

In previous sections, we have presented general discussions on the evaluation of tree-level CHY-like integrals, using the differential operator and the reduction matrix. This approach only hinges on the scattering equations and the factorized form of the integrand and therefore applies to all theories whose amplitudes admit the CHY representations. In this section, we consider a particular application of the method: the construction of the BCJ numerators for tree-level YangMills amplitudes.

\subsection{BCJ numerators from $\mathrm{CHY}$ form}

The CHY form [1-3] for a color-ordered amplitude in YangMills at tree level reads

$$
\begin{aligned}
& A(1, \alpha, n-1, n) \\
& \quad=\oint_{h_{1}=\cdots=h_{n-3}=0} d \Omega_{n} \sigma_{1 n-1 n}^{2} \operatorname{PT}(1 \alpha n-1 n) \operatorname{Pf}^{\prime}\left[\Psi_{1 n}(\sigma)\right],
\end{aligned}
$$

where $\alpha$ denotes a permutation of $\{2,3, \ldots, n-2\}$. Here we have used the shorthand notation $\sigma_{i_{1} \ldots i_{t}}=\left(\sigma_{i_{t}}-\right.$ $\left.\sigma_{i_{1}}\right) \prod_{j=1}^{t-1}\left(\sigma_{i_{j}}-\sigma_{i_{j+1}}\right)$ and introduced the measure

$d \Omega_{n}=\frac{J_{n}(\sigma) d \sigma_{2} \wedge \cdots \wedge d \sigma_{n-2}}{h_{1} \cdots h_{n-3}}$

Recall that $J_{n}(\sigma)=\prod_{1 \leq r<t \leq n-1}\left(\sigma_{t}-\sigma_{r}\right)$ denotes the Jacobian of the transformation to the polynomial scattering equations. We have adopted the gauge-fixing $\sigma_{1} \rightarrow 0, \sigma_{n-1} \rightarrow$
$1, \sigma_{n} \rightarrow \infty$ and $\sigma_{1 n-1 n}^{2}$ is the factor introduced after the gauge-fixing.

The Parke-Taylor factor corresponding to a given color ordering $(1, \alpha, n-1, n)$ reads

$\mathrm{PT}(1 \alpha n-1 n)=\frac{1}{\sigma_{1 \alpha(2) \ldots \alpha(n-2) n-1 n}}$.

$\operatorname{Pf}^{\prime}\left[\Psi_{1 n}(\sigma)\right]$ denotes the reduced Pfaffian of the matrix $\Psi(\sigma)$ with the first and the last columns and rows removed. The explicit expression for the reduced Pfaffian is given in [3] and there is a freedom of removing the $i$-th and $j$-th rows and columns of $\Psi$ for any $(i, j)$. Here for simplicity, we choose $(i, j)=(1, n)$.

On the other hand, the above color-ordered amplitudes is related to the BCJ numerators as follows,

$A(1, \alpha, n-1, n)=\sum_{\beta \in S_{n-3}} \mathbf{m}(\alpha \mid \beta) N(1 \beta n-1 n)$,

where $\mathbf{m}(\alpha \mid \beta)$ denotes the propagator matrix [45], whose rows and columns are labeled by the color orderings $(1, \alpha, n-1, n)$ and $(1, \beta, n-1, n)$. The BCJ numerators $N(1 \beta n-1 n)$ are in the minimal basis. Similar to the colorordered amplitude, the propagator matrix also admits a $\mathrm{CHY}$ like representation

$$
\begin{aligned}
& \mathbf{m}(\alpha \mid \beta) \\
& =-\oint_{h_{1}=\ldots=h_{n-3}=0} \sigma_{1 n-1 n}^{2} \operatorname{PT}(1 \alpha n-1 n) \operatorname{PT}(1 \beta n-1 n) d \Omega_{n} .
\end{aligned}
$$

Thus, comparing (47) and (51), we see that if $\operatorname{Pf}^{\prime}\left[\Psi_{1 n}(\sigma)\right]$ can be expanded in the basis spanned by the Parke-Taylor factors $\left\{\mathrm{PT}(1 \beta n-1 n) \mid \beta \in S_{n-2}\right\}$ with $\sigma$-independent coefficients, these coefficients are the BCJ numerators. To extract the BCJ numerators, we adopt a dual basis projector $\overline{\mathrm{PT}}(1 \alpha n-1 n)$ that satisfies the condition

$\oint_{h_{1}=\cdots=h_{n-3}=0}(-1) \sigma_{1 n-1 n}^{2} \overline{\mathrm{PT}}(1 \alpha n-1 n) \mathrm{PT}(1 \beta n-1 n) d \Omega_{n}=\delta_{\alpha \beta}$.

Recall that the propagator matrix $\mathbf{m}(\alpha \mid \beta)$ here is a $(n-3) ! \times$ $(n-3)$ ! square matrix. In this case, $\mathbf{m}(\alpha \mid \beta)$ is invertible and its inverse is the KLT matrix $\mathcal{S}_{\alpha \beta}[37,46,47]$. Thus the following is obviously the solution of the dual basis projector,

$\overline{\mathrm{PT}}(1 \alpha n-1 n)=\sum_{\beta} \mathcal{S}_{\alpha \beta} \mathrm{PT}(1 \beta n-1 n)$.

Using the dual basis, the BCJ numerator is given as a $\mathrm{CHY}$ like integral 


$$
\begin{aligned}
& N(1 \alpha n-1 n) \\
& =\oint_{h_{1}=\cdots=h_{n-3}=0} d \Omega_{n} \overline{\mathrm{PT}}(1 \alpha n-1 n) \sigma_{1 n-1 n}^{2} \operatorname{Pf}^{\prime}\left[\Psi_{1 n}(\sigma)\right],
\end{aligned}
$$

The CHY-representations of BCJ numerators can be easily evaluated using the differential-operator based method. Generally speaking, such BCJ numerators in the minimal basis are all non-local. They may contain poles that do not correspond to the propagators in trivalent diagrams. But these unphysical poles do not contribute to the amplitudes. As we will observe in examples, the numerators computed this way are gauge invariant in $n-2$ legs and respect the crossing symmetry under the permutation of $n-3$ legs.

\subsection{Yang-Mills BCJ numerator at four and five points}

In this section, we construct the BCJ numerators at four and five points to further illustrate the application of the differential operator and the reduction matrix. As discussed above, the BCJ numerator can be computed by a CHY-like integral, which is readily evaluated by our differential-operator based method.

At four points, the minimal basis consists of only one independent BCJ numerator, which we choose to be $N(1234)$. In this case, (54) gives

$$
N(1234)=\oint_{h_{1}=0} \sigma_{134}^{2} \overline{\mathrm{PT}}(1234) \mathrm{Pf}^{\prime}\left[\Psi_{14}(\sigma)\right] d \Omega_{4},
$$

where only one integration variable $\sigma_{2}$ is left after gauge fixing $\left(\sigma_{1} \rightarrow 0, \sigma_{3} \rightarrow 1, \sigma_{4} \rightarrow \infty\right)$. The KLT matrix is given in [37] and this leads to the dual basis projector below

$$
\overline{\mathrm{PT}}(1234)=\frac{s_{12} s_{1,23}}{s_{13}} \mathrm{PT}(1234) .
$$

The reduced Pfaffian can be written as the following [48]

$$
\begin{aligned}
\operatorname{Pf}^{\prime}\left[\Psi_{14}(\sigma)\right]= & -\frac{\epsilon_{1} \cdot F_{2} \cdot F_{3} \cdot \epsilon_{4}}{\sigma_{1234}}-\frac{\epsilon_{1} \cdot F_{3} \cdot F_{2} \cdot \epsilon_{4}}{\sigma_{1324}} \\
& +\frac{\epsilon_{1} \cdot \epsilon_{4} \operatorname{tr}\left(F_{2} \cdot F_{3}\right)}{2 \sigma_{14}^{2} \sigma_{23}^{2}}+\frac{\epsilon_{1} \cdot F_{2} \cdot \epsilon_{4}}{\sigma_{124}} C_{(3)} \\
& +\frac{\epsilon_{1} \cdot F_{3} \cdot \epsilon_{4}}{\sigma_{134}} C_{(2)}-\frac{\epsilon_{1} \cdot \epsilon_{4}}{\sigma_{14}^{2}} C_{(2)} C_{(3)},
\end{aligned}
$$

where we have adopted the following notations also used in [48]

$$
F_{i}=k_{i}^{\mu} \epsilon_{i}^{v}-\epsilon_{i}^{\mu} k_{i}^{v}, \quad C_{(i)}=\sum_{j=1, j \neq i}^{4} \frac{k_{j} \cdot \epsilon_{i}}{\sigma_{j}-\sigma_{i}} .
$$

We now demonstrate the evaluation of (55) using the reduction matrix. We consider the term below as an example and all other terms can be computed in the same way,

$N_{4} \equiv \oint_{h_{1}=\sigma_{0}-1=0} \sigma_{134}^{2} \overline{\mathrm{PT}}(1234) \frac{\epsilon_{1} \cdot F_{2} \cdot \epsilon_{4}}{\sigma_{124}} C_{(3)} d \Omega_{4}$

Plugging in (56) and homogenizing the scattering equations and the denominators, we have

$$
\begin{aligned}
N_{4}= & \frac{s_{12} s_{1,23} \epsilon_{1} \cdot F_{2} \cdot \epsilon_{4}}{s_{13}} \oint_{\substack{h_{1}=0 \\
\sigma_{0}-1=0}} \frac{d \sigma_{2} \wedge d \sigma_{0}}{\left(\sigma_{2}-\sigma_{0}\right)\left(\sigma_{0}-1\right)} \\
& \times\left(\frac{\epsilon_{3} \cdot k_{1}}{\sigma_{0}}-\frac{\epsilon_{3} \cdot k_{1}}{\sigma_{2}}-\frac{\epsilon_{3} \cdot k_{2}}{\sigma_{2}}\right) .
\end{aligned}
$$

Each term in the expression above equals to the action of a first-order differential operator, which can be written in terms of a common reduction matrix $M_{\sigma_{2}-\sigma_{0}}^{(4)}$ and the canonical coefficients of two prepared forms. That is,

$$
\begin{aligned}
N_{4}= & \frac{-s_{12} s_{1,23} \epsilon_{1} \cdot F_{2} \cdot \epsilon_{4}}{s_{13}} \\
& \times M_{\sigma_{2}-\sigma_{0}}^{(4)}\left(\epsilon_{3} \cdot k_{1} a_{0,0}^{\left(\sigma_{0}\right)}-\epsilon_{3} \cdot k_{1} a_{0,0}^{\left(\sigma_{2}\right)}-\epsilon_{3} \cdot k_{2} a_{0,0}^{\left(\sigma_{2}\right)}\right) \\
& \times\left.\left[\mathcal{D}_{1}^{(1)} \frac{1}{\sigma_{0}-1}\right]\right|_{\sigma \rightarrow 0},
\end{aligned}
$$

where $\mathcal{D}_{1}^{(1)}=\partial^{0,1}-\frac{s_{12}}{s_{13}} \partial^{1,0}, M_{\sigma_{2}-\sigma_{0}}^{(4)}$ has been computed previously in (33) and $a_{0,0}^{\left(\sigma_{r}\right)}$ is given by (26). Explicitly, they read

$M_{\sigma_{2}-\sigma_{0}}^{(4)}=-\frac{s_{13}}{s_{1,23}}, \quad a_{0,0}^{\left(\sigma_{2}\right)}=-\frac{1}{s_{12}}, \quad a_{0,0}^{\left(\sigma_{0}\right)}=\frac{1}{s_{13}}$.

Evaluating other terms in (57) similarly, we obtain the 4-point BCJ numerator in the minimal basis

$$
\begin{aligned}
N(1234)= & \epsilon_{1} \cdot F_{2} \cdot F_{3} \cdot \epsilon_{4}+\frac{s_{12}}{s_{13}} \epsilon_{1} \cdot F_{3} \cdot F_{2} \cdot \epsilon_{4} \\
& -\frac{s_{12}}{s_{1,23}} \operatorname{tr}\left(F_{2} \cdot F_{3}\right) \epsilon_{1} \cdot \epsilon_{4}-\frac{1}{s_{13}} k_{1} \cdot F_{3} \cdot k_{2} \epsilon_{1} \cdot F_{2} \cdot \epsilon_{4} \\
& -\frac{1}{s_{13}} k_{1} \cdot F_{2} \cdot k_{3} \epsilon_{1} \cdot F_{3} \cdot \epsilon_{4} \\
& -\frac{1}{s_{13} s_{1,23}} k_{1} \cdot F_{3} \cdot k_{2} k_{1} \cdot F_{2} \cdot k_{3} \epsilon_{1} \cdot \epsilon_{4}
\end{aligned}
$$

As $F_{i}$ vanishes under the gauge transformation $\epsilon_{i} \rightarrow k_{i}$, $N$ (1234) is manifestly gauge invariant in leg 2 and leg 3.

At five points, the minimal basis consists of two independent BCJ numerators $N(12345)$ and $N(13245)$. As pointed out before, the numerators computed this way is crossing 
symmetric and therefore it suffices to compute $N(12345)$ only. This numerator reads

$$
N(12345)=\oint_{h_{1}=h_{2}=0} \sigma_{145}^{2} \overline{\mathrm{PT}}(12345) \mathrm{Pf}^{\prime}\left[\Psi_{15}(\sigma)\right] d \Omega_{5} .
$$

where the dual basis projector $\overline{\mathrm{PT}}(12345)$ can be easily computed from (53) and takes the form below

$$
\begin{aligned}
\overline{\mathrm{PT}}(12345)= & \left(\frac{s_{12} s_{34} s_{1,24} s_{12,3}}{s_{14} s_{124}}+\frac{s_{12}^{2} s_{34} s_{13}}{s_{14} s_{134}}\right) \mathrm{PT}(12345) \\
& +\left(\frac{s_{12} s_{13} s_{24} s_{34}}{s_{14} s_{134}}+\frac{s_{12} s_{13} s_{24} s_{34}}{s_{14} s_{124}}\right) \mathrm{PT}(13245) .
\end{aligned}
$$

inators above are homogenized with an auxiliary variable $\sigma_{0}$. This process leads to

$$
\begin{gathered}
C_{(4)=} \frac{s_{14}}{-\sigma_{0}}+\frac{s_{24}}{\sigma_{2}-\sigma_{0}}+\frac{s_{34}}{\sigma_{3}-\sigma_{0}}, \\
\frac{1}{\sigma_{1235}}=\frac{1}{\sigma_{2}\left(\sigma_{2}-\sigma_{3}\right)} .
\end{gathered}
$$

There are no other denominators in (67) after gauge fixing. The factor $C_{(4)}$ can now be replaced by a matrix denoted as $M_{C_{(4)}}^{(5)}$, which is a sum over three reduction matrices, each corresponding to the denominator of one of its three terms. These reduction matrices can all be constructed following the discussions in the previous section. This matrix $M_{C_{(4)}}^{(5)}$ reads

$M_{C_{(4)}}^{(5)} \equiv\left(\begin{array}{cc}\frac{-s_{1,34} s_{124} k_{2} \cdot F_{4} \cdot k_{3}+s_{234} s_{34} k_{1} \cdot F_{4} \cdot k_{2}}{s_{234} s_{24} s_{34}} & -\frac{s_{13} s_{134} k_{2} \cdot F_{4} \cdot k_{3}}{s_{24} s_{24} s_{34}} \\ -\frac{s_{12} s_{124} k_{3} \cdot F_{4} \cdot k_{2}}{s_{234} s_{24} s_{34}} & \frac{-s_{1,24} s_{134} k_{3} \cdot F_{4} \cdot k_{2}+s_{234} s_{24} k_{1} \cdot F_{4} \cdot k_{3}}{s_{234} s_{24} s_{34}}\end{array}\right)$

The reduced Pfaffian at five points reads [48]

$$
\begin{aligned}
\operatorname{Pf}^{\prime}[ & \left.\Psi_{15}(\sigma)\right] \\
= & \sum_{\gamma \in S_{3}}\left(-\frac{\epsilon_{1} \cdot F_{\gamma(2)} \cdot F_{\gamma(3)} \cdot F_{\gamma(4)} \cdot \epsilon_{5}}{\sigma_{1 \gamma(2) \gamma(3) \gamma(4) 5}}\right. \\
& \left.+\frac{\epsilon_{1} \cdot F_{\gamma(2)} \cdot \epsilon_{5} \operatorname{tr}\left(F_{\gamma(3)} F_{\gamma(4)}\right)}{4 \sigma_{1 \gamma(2) 5} \sigma_{\gamma(3) \gamma(4)}}\right)+\frac{\epsilon_{1} \cdot \epsilon_{5} \operatorname{tr}\left(F_{2} F_{3} F_{4}\right)}{2 \sigma_{15} \sigma_{234}} \\
& +\frac{\epsilon_{1} \cdot \epsilon_{5} \operatorname{tr}\left(F_{3} F_{2} F_{4}\right)}{2 \sigma_{15} \sigma_{324}}+\sum_{\gamma \in S_{3}}\left(\frac{\epsilon_{1} \cdot F_{\gamma(2)} \cdot F_{\gamma(3)} \cdot \epsilon_{5} C_{(\gamma(4))}}{\sigma_{1 \gamma(2) \gamma(3) 5}}\right. \\
& \left.-\frac{\epsilon_{1} \cdot F_{\gamma(2)} \cdot \epsilon_{5} C_{(\gamma(3))} C_{(\gamma(4))}}{2 \sigma_{1 \gamma(2) 5}}\right) \\
& -\epsilon_{1} \cdot \epsilon_{5}\left(\frac{\operatorname{tr}\left(F_{2} F_{3}\right) C_{(4)}}{\sigma_{15} \sigma_{23}}+\frac{C_{(2)} \operatorname{tr}\left(F_{3} F_{4}\right)}{\sigma_{15} \sigma_{34}}\right. \\
& \left.+\frac{\operatorname{tr}\left(F_{2} F_{4}\right) C_{(3)}}{\sigma_{15} \sigma_{24}}-\frac{C_{(2)} C_{(3)} C_{(4)}}{\sigma_{15}}\right) .
\end{aligned}
$$

Like the four-point case, we also consider the characteristic term below as an example here and the rest can all be computed in the same way,

$N_{5}=\oint_{h_{1}=h_{2}=0} \sigma_{145}^{2} \overline{\mathrm{PT}}(12345) \frac{\epsilon_{1} \cdot F_{2} \cdot F_{3} \cdot \epsilon_{5} C_{(4)}}{\sigma_{1235}} d \Omega_{5}$.

Taking $\sigma_{1} \rightarrow 0, \sigma_{4} \rightarrow 1, \sigma_{5} \rightarrow \infty$, we are only left with two variables $\sigma_{2}$ and $\sigma_{3}$. Thescattering equations and the denom-
Another common reduction matrix $M_{\sigma_{2}-\sigma_{3}}^{(5)}$ renders (67) a prepared form. This reduction matrix reads

$$
M_{\sigma_{2}-\sigma_{3}}^{(5)}=\left(\begin{array}{cc}
\frac{\left(s_{12} s_{14}+s_{1,34} s_{23}\right) s_{124}}{s_{1,231} s_{23} s_{123}} & \frac{s_{13}\left(s_{14}-s_{23}\right) s_{134,2}}{s_{1,234} s_{23} s_{123}} \\
\frac{s_{12}\left(s_{14}-s_{23}\right) s_{124}}{s_{1,234} s_{23} s_{123}} & \frac{\left(s_{13} s_{14}+s_{1,24} s_{23}\right) s_{134,2}}{s_{1,234} s_{23} s_{123}}
\end{array}\right) .
$$

The canonical $a$-coefficients for the prepared form with $h_{0}=$ $\sigma_{2}$ are

$a_{010}^{\left(\sigma_{2}\right)}=\frac{1}{s_{12} s_{124}}, \quad a_{100}^{\left(\sigma_{2}\right)}=0$

Hence (67) is given by the action of the differential operator as follows

$$
\begin{aligned}
N_{5}= & -\frac{\epsilon_{1} \cdot F_{2} \cdot F_{3} \cdot \epsilon_{5}}{2} \sum_{i, j=1}^{2}\left(M_{C_{(4)}}^{(5)} M_{\sigma_{2}-\sigma_{3}}^{(5)}\right)_{i, j} a_{\gamma_{i}(0), \gamma_{i}(1), 0}^{\left(\sigma_{2}\right)} \\
& \times\left.\left[\mathcal{D}_{j}^{(3)} \frac{\sigma_{145} \overline{\mathrm{PT}}(12345) J_{5}(\sigma)}{\left(\sigma_{0}-1\right)}\right]\right|_{\sigma \rightarrow 0},
\end{aligned}
$$

where $\mathcal{D}_{j}^{(3)}$ is given in (41) and $\gamma_{i} \in S_{2}$. Plugging in the expressions (41), (69), (70) and (71), we obtain

$N_{5}=\frac{k_{1} \cdot F_{4} \cdot k_{3}}{s_{14}} \epsilon_{1} \cdot F_{2} \cdot F_{3} \cdot \epsilon_{5}-\frac{s_{34} k_{1} \cdot F_{4} \cdot k_{2}}{s_{14} s_{124}} \epsilon_{1} \cdot F_{2} \cdot F_{3} \cdot \epsilon_{5}$. 
All other terms in (66) are treated similarly and the explicit expression for the five-point BCJ numerator reads

$$
\begin{aligned}
& N(12345)=\left(k_{1} \cdot F_{4} \cdot k_{3}-\frac{s_{34}}{s_{124}} k_{1} \cdot F_{4} \cdot k_{2}\right) \frac{\epsilon_{1} \cdot F_{2} \cdot F_{3} \cdot \epsilon_{5}}{s_{14}} \\
& +\frac{1}{s_{14}} k_{1} \cdot F_{2} \cdot F_{3} \cdot F_{4} \cdot k_{1} \epsilon_{1} \cdot \epsilon_{5} \\
& +\left(\frac{1}{s_{134}} k_{1} \cdot F_{2} \cdot k_{34} k_{1} \cdot F_{3} \cdot F_{4} \cdot k_{1}\right. \\
& \left.+\frac{1}{s_{124}} k_{12} \cdot F_{3} \cdot k_{4} k_{1} \cdot F_{2} \cdot F_{4} \cdot k_{1}\right) \frac{\epsilon_{1} \cdot \epsilon_{5}}{s_{14}} \\
& +\left(\frac{s_{12} s_{23}+s_{14} s_{2,34}}{s_{124} s_{14} s_{134}} k_{1} \cdot F_{3} \cdot F_{4} \cdot k_{1}\right. \\
& \left.+\frac{s_{12} s_{23}+s_{12,3} s_{2,34}}{s_{25} s_{34}} \operatorname{tr}\left(F_{3} \cdot F_{4}\right)\right) \epsilon_{1} \cdot F_{2} \cdot \epsilon_{5} \\
& -\left(k_{1} \cdot F_{3} \cdot F_{4} \cdot k_{2}+\frac{s_{1,24}}{s_{14}} k_{2} \cdot F_{3} \cdot F_{4} \cdot k_{1}\right. \\
& \left.+k_{2} \cdot F_{3} \cdot F_{4} \cdot k_{2}\right) \frac{\epsilon_{1} \cdot F_{2} \cdot \epsilon_{5}}{s_{124}} \\
& +\left(\frac{\left(s_{14}-s_{23}\right) s_{34}}{s_{124} s_{14}} k_{1} \cdot F_{2} \cdot F_{4} \cdot k_{1}-k_{1} \cdot F_{2} \cdot F_{4} \cdot k_{3}\right. \\
& \left.-\frac{1}{s_{14}} k_{1} \cdot F_{2} \cdot k_{3} k_{1} \cdot F_{4} \cdot k_{3}\right) \frac{\epsilon_{1} \cdot F_{3} \cdot \epsilon_{5}}{s_{134}} \\
& +\left(k_{1} \cdot F_{2} \cdot F_{3} \cdot k_{4}+\frac{s_{12}}{s_{14}} k_{14} \cdot F_{2} \cdot F_{3} \cdot k_{4}\right. \\
& \left.-\frac{s_{1,24}}{s_{14}} k_{1} \cdot F_{2} \cdot k_{3} k_{1} \cdot F_{3} \cdot k_{4}\right) \frac{\epsilon_{1} \cdot F_{4} \cdot \epsilon_{5}}{s_{124}} \\
& +\left(\frac{s_{12}}{s_{14} s_{134}} k_{3} \cdot F_{2} \cdot k_{4} k_{1} \cdot F_{3} \cdot k_{4}\right. \\
& \left.-\frac{1}{s_{134}} k_{1} \cdot F_{2} \cdot k_{4} k_{1} \cdot F_{3} \cdot k_{4}\right) \frac{\epsilon_{1} \cdot F_{4} \cdot \epsilon_{5}}{s_{124}} \\
& +\frac{s_{12}}{s_{14} s_{134}} k_{1} \cdot F_{4} \cdot k_{3} \epsilon_{1} \cdot F_{3} \cdot F_{2} \cdot \epsilon_{5} \\
& +\frac{1}{s_{124}} k_{12} \cdot F_{3} \cdot k_{4} \epsilon_{1} \cdot F_{2} \cdot F_{4} \cdot \epsilon_{5} \\
& +\left(\frac{\left(s_{14}-s_{23}\right)}{s_{134}} k_{1} \cdot F_{3} \cdot k_{4}+k_{2} \cdot F_{3} \cdot k_{4}\right) \frac{s_{12} \epsilon_{1} \cdot F_{4} \cdot F_{2} \cdot \epsilon_{5}}{s_{14} s_{124}} \\
& -\left(\frac{s_{1,24} s_{13,4}}{s_{14}} k_{1} \cdot F_{2} \cdot k_{3}-s_{13} k_{1} \cdot F_{2} \cdot k_{4}\right. \\
& \left.-\frac{s_{12} s_{13,4}}{s_{14}} k_{3} \cdot F_{2} \cdot k_{4}\right) \frac{\epsilon_{1} \cdot F_{4} \cdot F_{3} \cdot \epsilon_{5}}{s_{124} s_{134}} \\
& +\frac{1}{s_{134}} k_{1} \cdot F_{2} \cdot k_{34} \epsilon_{1} \cdot F_{3} \cdot F_{4} \cdot \epsilon_{5}-\epsilon_{1} \cdot F_{2} \cdot F_{3} \cdot F_{4} \cdot \epsilon_{5} \\
& -\frac{s_{12,3}}{s_{124}} \epsilon_{1} \cdot F_{2} \cdot F_{4} \cdot F_{3} \cdot \epsilon_{5} \\
& -\frac{s_{12}}{s_{134}} \epsilon_{1} \cdot F_{3} \cdot F_{4} \cdot F_{2} \cdot \epsilon_{5}+\frac{s_{12} s_{34}}{s_{124} s_{14}} \epsilon_{1} \cdot F_{4} \cdot F_{2} \cdot F_{3} \cdot \epsilon_{5} \\
& +\frac{s_{12} s_{13,4}}{s_{134} s_{14}} \epsilon_{1} \cdot F_{4} \cdot F_{3} \cdot F_{2} \cdot \epsilon_{5} \text {. }
\end{aligned}
$$

The other numerator is obtained by the permuting indices

$$
N(13245)=\left.N(12345)\right|_{2 \leftrightarrow 3} .
$$

These non-local BCJ numerators are compact and again manifestly gauge invariant in the leg 2,3 and 4, due to the existence of the $F_{i}$ factors.

\section{Conclusion and outlooks}

In this paper, we have developed a systematic method to construct the BCJ numerators, starting from the CHY forms of scattering amplitudes and using the differential operator proposed in our previous work [23]. This method is based on the key observation that the number of canonical coefficients in such a differential operator is always $(n-3)$ ! for a $n$-point CHY form, independent of the order of the operator. In the process of solving for the canonical coefficients, we have built the reduction matrices to simplify the differential operator and improve the efficiency of computation. The reduction matrices are also universal for all the theories. In the end, as we have demonstrated, we always arrive at the prepared forms for which the coefficients are solved analytically in [24].

Both the BCJ numerators and the amplitudes obtained this way enjoy the manifest gauge invariance in $(n-2)$ out of the $n$ external legs, and their final expressions are always of factorized forms. It is hopeful to formulate a closed form for the reduction matrix in future works, since a given reduction matrix $M_{\sigma_{i}}$ is determined only by the factor $\sigma_{i}$ and the polynomial scattering equations. Moreover, the polynomial scattering equations have nice combinatoric structures to exploit, which may allow us to produce analytical results for a general reduction matrix. As discussed in [21], the polynomial scattering equations is a Macaulay H-basis. This property may be helpful to prove the observation of the number $(n-3)$ ! of independent $a$-coefficients.

In this paper, the concept of dual basis is adopted to extract the non-local BCJ numerators in the minimal basis, in which the non-local propagators can be removed using the $\mathrm{BCJ}$ relations. It is conceivable that similar projectors can be constructed for the local BCJ numerators as well. It can be expected that such projectors are constructed recursively, which may point to novel algebraic structures [49-52].

Our method can be easily generalized to loop levels. The one-loop scattering equations and the prepared forms have been studied in [24]. We expect the canonical coefficients can also be found at one loop and then the construction of reduction matrix is expected to be straightforward. Another future direction is to carry our method over to string theory. String amplitudes, written in the forms of worldsheet integrals, have a number of features in common with the CHYlike integrals. It is reasonable to hope that there exist similar differential operators and even reduction matrices which can help us evaluate those worldsheet integrals efficiently while preserving the factorized form. 
Acknowledgements G.C. and T.W. thank H. Johansson for useful discussion and kind suggestions. The research of G.C. is supported in part by the Knut and Alice Wallenberg Foundation under grant KAW 2013.0235, and the Ragnar Söderberg Foundation under grant S1/16. G.C. has been supported in parts by NSF of China Grant under Contract 11405084, the Open Project Program of State Key Laboratory of Theoretical Physics, Institute of Theoretical Physics, Chinese Academy of Sciences (No.Y5KF171CJ1). T.W. thanks the China Scholarship Council for support (File No. 201706190098).

Data Availability Statement This manuscript has no associated data or the data will not be deposited. [Authors' comment: The result of this paper is based on the theoretical analysis and mathematical computation. So there is no data from experiments.]

Open Access This article is licensed under a Creative Commons Attribution 4.0 International License, which permits use, sharing, adaptation, distribution and reproduction in any medium or format, as long as you give appropriate credit to the original author(s) and the source, provide a link to the Creative Commons licence, and indicate if changes were made. The images or other third party material in this article are included in the article's Creative Commons licence, unless indicated otherwise in a credit line to the material. If material is not included in the article's Creative Commons licence and your intended use is not permitted by statutory regulation or exceeds the permitted use, you will need to obtain permission directly from the copyright holder. To view a copy of this licence, visit http://creativecomm ons.org/licenses/by/4.0/.

Funded by SCOAP ${ }^{3}$.

\section{References}

1. F. Cachazo, S. He, E.Y. Yuan, Phys. Rev. Lett. 113(17), 171601 (2014). https://doi.org/10.1103/PhysRevLett.113.171601

2. F. Cachazo, S. He, E.Y. Yuan, JHEP 07, 033 (2014). https://doi. org/10.1007/JHEP07(2014)033

3. F. Cachazo, S. He, E.Y. Yuan, JHEP 01, 121 (2015). https://doi. org/10.1007/JHEP01(2015)121

4. E. Casali, Y. Geyer, L. Mason, R. Monteiro, K.A. Roehrig, JHEP 11, 038 (2015). https://doi.org/10.1007/JHEP11(2015)038

5. Y. Geyer, L. Mason, R. Monteiro, P. Tourkine, Phys. Rev. Lett. 115(12), 121603 (2015). https://doi.org/10.1103/PhysRevLett. 115.121603

6. Y. Geyer, L. Mason, R. Monteiro, P. Tourkine, JHEP 03, 114 (2016). https://doi.org/10.1007/JHEP03(2016)114

7. Y. Geyer, L. Mason, R. Monteiro, P. Tourkine, Phys. Rev. D 94(12), 125029 (2016). https://doi.org/10.1103/PhysRevD.94.125029

8. F. Cachazo, S. He, E.Y. Yuan, JHEP 08, 008 (2016). https://doi. org/10.1007/JHEP08(2016)008

9. S. He, E.Y. Yuan, Phys. Rev. D 92(10), 105004 (2015). https://doi. org/10.1103/PhysRevD.92.105004

10. B. Feng, JHEP 05, 061 (2016). https://doi.org/10.1007/ JHEP05(2016)061

11. H. Gomez, Phys. Rev. D 95(10), 106006 (2017). https://doi.org/ 10.1103/PhysRevD.95.106006

12. H. Gomez, C. Lopez-Arcos, P. Talavera, JHEP 10, 175 (2017). https://doi.org/10.1007/JHEP10(2017)175

13. S. He, O. Schlotterer, Y. Zhang, Nucl. Phys. B 930, 328 (2018). https://doi.org/10.1016/j.nuclphysb.2018.03.003

14. L. Dolan, P. Goddard, JHEP 05, 010 (2014). https://doi.org/10. 1007/JHEP05(2014)010

15. L. Dolan, P. Goddard, JHEP 07, 029 (2014). https://doi.org/10. 1007/JHEP07(2014)029
16. C. Baadsgaard, N.E.J. Bjerrum-Bohr, J.L. Bourjaily, P.H. Damgaard, JHEP 09, 129 (2015). https://doi.org/10.1007/ JHEP09(2015)129

17. C. Baadsgaard, N.E.J. Bjerrum-Bohr, J.L. Bourjaily, P.H. Damgaard, B. Feng, JHEP 11, 080 (2015). https://doi.org/10.1007/ JHEP11(2015)080

18. C. Cardona, B. Feng, H. Gomez, R. Huang, JHEP 09, 133 (2016). https://doi.org/10.1007/JHEP09(2016)133

19. R. Huang, Y.J. Du, B. Feng, JHEP 06, 133 (2017). https://doi.org/ 10.1007/JHEP06(2017)133

20. M. Sogaard, Y.Zhang, Phys. Rev. D 93(10), 105009 (2016). https:// doi.org/10.1103/PhysRevD.93.105009

21. J. Bosma, M. Sogaard, Y. Zhang, Phys. Rev. D 94(4), 041701 (2016). https://doi.org/10.1103/PhysRevD.94.041701

22. K.J. Larsen, R. Rietkerk, Comput. Phys. Commun. 222, 250 (2018). https://doi.org/10.1016/j.cpc.2017.08.025

23. T. Wang, G. Chen, Y.K.E. Cheung, F. Xu, JHEP 01, 028 (2017). https://doi.org/10.1007/JHEP01(2017)028

24. T. Wang, G. Chen, Y.K.E. Cheung, F. Xu, JHEP 06, 015 (2017). https://doi.org/10.1007/JHEP06(2017)015

25. Z. Bern, J.J.M. Carrasco, H. Johansson, Phys. Rev. D 78, 085011 (2008). https://doi.org/10.1103/PhysRevD.78.085011

26. R. Monteiro, D. O'Connell, JHEP 07, 007 (2011). https://doi.org/ 10.1007/JHEP07(2011)007

27. J. Broedel, L.J. Dixon, JHEP 10, 091 (2012). https://doi.org/10. 1007/JHEP10(2012)091

28. T. Bargheer, S. He, T. McLoughlin, Phys. Rev. Lett. 108, 231601 (2012). https://doi.org/10.1103/PhysRevLett.108.231601

29. A. Ochirov, P. Tourkine, JHEP 05, 136 (2014). https://doi.org/10. 1007/JHEP05(2014)136

30. M. Chiodaroli, Q. Jin, R. Roiban, JHEP 01, 152 (2014). https://doi. org/10.1007/JHEP01(2014)152

31. S. Stieberger, T.R. Taylor, Phys. Lett. B 739, 457 (2014). https:// doi.org/10.1016/j.physletb.2014.10.057

32. H. Johansson, A. Ochirov, JHEP 11, 046 (2015). https://doi.org/ 10.1007/JHEP11(2015)046

33. M. Chiodaroli, M. Gnaydin, H. Johansson, R. Roiban, JHEP 01, 081 (2015). https://doi.org/10.1007/JHEP01(2015)081

34. Yt Huang, H. Johansson, S. Lee, JHEP 11, 050 (2013). https://doi. org/10.1007/JHEP11(2013)050

35. G. Chen, Y.J. Du, JHEP 01, 061 (2014). https://doi.org/10.1007/ JHEP01(2014)061

36. G. Chen, Y.J. Du, S. Li, H. Liu, JHEP 03, 156 (2015). https://doi. org/10.1007/JHEP03(2015)156

37. N.E.J. Bjerrum-Bohr, P.H. Damgaard, T. Sondergaard, P. Vanhove, JHEP 01, 001 (2011). https://doi.org/10.1007/JHEP01(2011)001

38. C.R. Mafra, O. Schlotterer, S. Stieberger, JHEP 07, 092 (2011). https://doi.org/10.1007/JHEP07(2011)092

39. C.H. Fu, Y.J. Du, B. Feng, JHEP 03, 050 (2013). https://doi.org/ 10.1007/JHEP03(2013)050

40. C.R. Mafra, O. Schlotterer, JHEP 03, 097 (2016). https://doi.org/ 10.1007/JHEP03(2016)097

41. N.E.J. Bjerrum-Bohr, J.L. Bourjaily, P.H. Damgaard, B. Feng, JHEP 09, 094 (2016). https://doi.org/10.1007/JHEP09(2016)094

42. C.R. Mafra, JHEP 07, 080 (2016). https://doi.org/10.1007/ JHEP07(2016)080

43. Y.J. Du, F. Teng, JHEP 04, 033 (2017). https://doi.org/10.1007/ JHEP04(2017)033

44. R. Hartshorne, Algebraic geometry, vol. 52 (Springer Science \& Business Media, Berlin, 2013)

45. D. Vaman, Y.P. Yao, J. High Energy Phys. 2010(11), 28 (2010). https://doi.org/10.1007/JHEP11(2010)028

46. H. Kawai, D.C. Lewellen, S.H.H. Tye, Nucl. Phys. B 269, 1 (1986) https://doi.org/10.1016/0550-3213(86)90362-7

47. Z. Bern, L.J. Dixon, M. Perelstein, J.S. Rozowsky, Nucl. Phys. B 546, 423 (1999). https://doi.org/10.1016/S0550-3213(99)00029-2 
48. C.S. Lam, Y.P. Yao, Phys. Rev. D 93(10), 105008 (2016). https:// doi.org/10.1103/PhysRevD.93.105008

49. V. Drinfeld, Leningrad Math. J. 1, 1419 (1990). https://doi.org/10. 1007/JHEP03(2013)050

50. S. Dascalescu, C. Nastasescu, S. Raianu, Hopf algebra: An introduction (CRC Press, London, 2000)
51. C.H. Fu, K. Krasnov, JHEP 01, 075 (2017). https://doi.org/10. 1007/JHEP01(2017)075

52. C. Duhr, JHEP 08, 043 (2012). https://doi.org/10.1007/ JHEP08(2012)043 